\title{
ASPECTS OF TWO-SIDED SURFACE WAVINESS IN AN EHL LINE CONTACT
}

\author{
A.A. Lubrecht ${ }^{a, b}$ and C.H. Venner ${ }^{b}$ \\ ${ }^{a}$ SKF Engineering \& Research Centre B.V., Nieuwegein, the Netherlands \\ ${ }^{b}$ University of Twente, Enschede, The Netherlands
}

Applying a multigrid solver to the transient Reynolds equation, this paper investigates the influence of two-sided waviness on pressure and film thickness in a medium loaded EHL line contact. The contact properties are studied for one set of operating conditions varying the ratio of the surface velocities, as well as the amplitude and wavelength of the waviness. The characteristic quantities used to describe the transient problem are straightforward extensions of the ones describing the stationary problem such as $H_{\min }, H_{\text {ave }}$ and $P_{\max }$. Where possible physical explanations of the observed relations are presented.

\section{Introduction}

The research in the field of numerical Elasto Hydrodynamic Lubrication (EHL) is gradually moving away from the 'classical' idealized smooth surface problem and facing the complex reality in which engineering surfaces are non-smooth on the scale of thickness of the lubricating oil film. Presently we are puzzled by a problem that must have sounded familiar to a tribologist in the early decades of this century: How to understand, utilize and optimize the separating oil film in highly loaded contacts. Whereas the previous problem focussed on the so-called macro contact, the current one deals with the micro contact, the influence of surface roughness. The goal is to predict and optimize the operation of highly loaded contacts working with film thicknesses smaller than the undeformed surface roughness amplitude.

A number of investigations have addressed the steady state roughness problem $[5,6,7,9,10,12]$ and [13], however in general the roughness moves through the contact, thereby creating a transient problem. The time dependent problem was addressed in $[1,2,3,14,17]$. In [11] the transient analysis was used to explain an experimentally observed sensitivity to the overrolling direction of the location of the failure site with respect to the original indentation. In [19] a transient analysis of the influence of one-sided waviness on the film thickness was described. Several unexpected features were found, related to the velocity at which pressure and film thickness perturbations travel through the contact zone. It was shown that the pressure perturbations travel with the speed of the wavy surface ( $u_{2}$ in that case), whereas the film thickness disturbances move with the average surface speed $\left(u_{1}+u_{2}\right) / 2$. These predicted features have recently been observed experimentally [8]. As was shown in the theoretical paper [19] the solution of the general non-smooth surface contact problem requires a transient solution of the EHL equations. In that paper as well as in the present one the authors have selected Multigrid solvers, since they meet the two requirements necessary to tackle this particular problem; they are fast and robust. Furthermore, and perhaps the most important argument is, that these techniques can straightforwardly be extended to more complete (and thus more complex) models of reality as for instance the point contact problem. This extension of the algorithm to point contact problems causes the computational complexity to increase only moderately. As a consequence the increase in computing time is not a large obstacle in view of the present increase in computer capacity. The main reason for addressing the line contact problem in this work is not so much the gain in computing time as the additional simplicity of the line contact problem when compared 
with the full non-smooth point contact problem.

In this paper the waviness extends to both surfaces, which introduces two additional degrees of freedom to the problem compared to the case studied in [19]. The complete problem is described by the contact condition parameters $(M, L$ or $W, U, G)$, the ratio of the surface velocities and for sinusoidal waviness the dimensionless amplitude and wavelength of both surfaces, and the initial phase difference. To reduce the number of parameters to some extent, the waviness wavelength on the upper and lower surface is assumed identical. By calculating either average values or extreme values over time, i.e. averages/extremes over all phase differences, the initial phase difference of the waviness becomes irrelevant.

\section{$1.1 \quad$ Notation}

$A$

dimensionless amplitude, $\mathcal{A}=A R / b^{2}$

$b$ halfwidth of Hertzian contact,

$b=\sqrt{(8 \mathrm{w} R) /\left(\pi E^{\prime}\right)}$

$E \quad$ modulus of elasticity

$E^{\prime} \quad$ reduced modulus of elasticity, $2 / E^{\prime}=\left(1-\nu_{1}^{2}\right) / E_{1}+\left(1-\nu_{2}^{2}\right) / E_{2}$

$G$ dimensionless materials parameter, $G=\alpha E^{\prime}$

$h \quad$ film thickness

$h_{\text {ave }}$ average film thickness, $h_{\text {ave }}=\frac{1}{2 b} \int_{-b}^{+b} h(x) d x$

$h_{\text {inv }}$ inverse film thickness, $h_{i n v}=\left(\frac{1}{x_{b}-x_{\mathrm{a}}} \int_{x_{\mathrm{a}}}^{x_{\mathrm{b}}} h^{-1}(x) d x\right)^{-1}$

$h_{\min }$ minimum film thickness

$H$ dimensionless film thickness, $H=h R / b^{2}$

$H_{\text {ave }}$ dimensionless average film thickness, $H_{\text {ave }}=h_{\text {ave }} R / b^{2}$

$H_{a v e}^{+} \quad$ maximum over time of $H_{a v e}$

$H_{\text {ave }}^{-} \quad$ minimum over time of $H_{\text {ave }}$

$\overline{H_{\text {ave }}}$ average over time of $H_{\text {ave }}$

$H_{\text {inv }}$ dimensionless inverse film thickness,

$H_{\text {inv }}=h_{\text {inv }} R / b^{2}$

$\overline{H_{i n v}}$ average over time of $H_{i n v}$

$H_{\min }$ dimensionless minimum film thickness, $H_{\min }=h_{\min } R / b^{2}$

$H_{\min }^{+} \quad$ maximum over time of $H_{\min }$

$H_{m i n}^{-}$minimum over time of $H_{\min }$

$H_{0} \quad$ integration constant

$L$ dimensionless material parameter (Moes),
$L=G(2 U)^{0.25}$

$M$ dimensionless load parameter (Moes), $M=W(2 U)^{-0.5}$

$n \quad$ number of discretisation points

$p$ pressure

$p_{h} \quad$ maximum Hertzian pressure, $p_{h}=(2 \mathrm{w}) /(\pi b)$

$P \quad$ dimensionless pressure, $P=p / p_{h}$

$P_{\max }$ maximum dimensionless pressure

$P_{\max }^{+} \quad$ maximum over time of $P_{\max }$

$P_{\max }^{-} \quad$ minimum over time of $P_{\max }$

$R \quad$ reduced radius of curvature, $1 / R=1 / R_{1}+1 / R_{2}$

$\mathcal{R}$ dimensionless deviations from the smooth profile

$t$ time

$T \quad$ dimensionless time, $T=\left(t u_{s}\right) /(2 b)$

$u_{1} \quad$ velocity of lower surface

$u_{2} \quad$ velocity of upper surface

$u_{s} \quad$ sum velocity, $u_{s}=u_{1}+u_{2}$

$U$ dimensionless speed parameter, $U=\eta_{0} u_{s} /\left(2 E^{\prime} R\right)$

$x \quad$ coordinate

$x_{d} \quad$ location of surface feature

$x_{3}$ location of surface feature at $t=0$

$X, X^{\prime}$ dimensionless coordinate, $X=x / b, X^{\prime}=x^{\prime} / b$

$X_{d}$ dimensionless location of surface feature, $X_{d}=x_{d} / b$

$X$ dimensionless location of surface feature at $T=0, X_{s}=x_{s} / b$

$X_{a}, X_{b}$ dimensionless inlet, outlet boundary domain, $X_{a}=x_{a} / b, X_{b}=x_{b} / b$

w external load per unit width

$W$ dimensionless load parameter, $W=\mathrm{w} /\left(E^{\prime} R\right)$

$w \quad$ wavelength of surface feature

$\mathcal{W}$ dimensionless wavelength of surface feature, $\mathcal{W}=w / b$

$z \quad$ viscosity index (Roelands equation)

$\alpha \quad$ pressure viscosity index

$\bar{\alpha} \quad$ dimensionless parameter, $\bar{\alpha}=\alpha p_{h}$

$\Delta_{T} \quad$ dimensionless time increment

$\Delta_{X} \quad$ dimensionless space increment

$\epsilon$ coefficient in Reynolds equation, $\epsilon=\left(\bar{\rho} H^{3}\right) /(\bar{\eta} \lambda)$

$\lambda$ dimensionless speed parameter, $\lambda=\left(6 \eta_{0} u_{s} R^{2}\right) /\left(b^{3} p_{h}\right)$

$\eta \quad$ viscosity 
$\eta_{0} \quad$ viscosity at ambient pressure

$\bar{\eta} \quad$ dimensionless viscosity, $\bar{\eta}=\eta / \eta_{0}$

$\rho$ density

$\rho_{0} \quad$ density at ambient pressure

$\bar{\rho} \quad$ dimensionless density, $\bar{\rho}=\rho / \rho_{0}$

\section{Theory}

For completeness this section first presents the equations to be solved. Subsequently, the physical parameters describing the contact conditions are given in Table 1, together with different sets of dimensionless parameters. Table 2 lists the numerical parameters used in the calculations. The theory behind the Multigrid solvers is not repeated here and can be found in for instance $[12,16,18]$, and in the references of these works.

\subsection{Equations}

The one-dimensional transient Reynolds equation is written in a dimensionless form:

$$
\frac{\partial}{\partial X}\left(\epsilon \frac{\partial P}{\partial X}\right)-\frac{\partial(\bar{\rho} H)}{\partial X}-\frac{\partial(\bar{\rho} H)}{\partial T}=0
$$

The boundary conditions are $P\left(X_{a}, T\right)=$ $P\left(X_{b}, T\right)=0, \forall T$ where $X_{a}$ and $X_{b}$ denote the boundaries of the domain. Furthermore, the cavitation condition $P(X, T) \geq 0, \forall X, T$ must be satisfied throughout the domain. $\epsilon$ and $\lambda$ are defined according to:

$$
\epsilon=\frac{\bar{\rho} H^{3}}{\bar{\eta} \lambda} \quad \lambda=\frac{6 \eta_{0} u_{s} R^{2}}{b^{3} p_{h}}
$$

The density $\rho$ is assumed to depend on the pressure according to the Dowson and Higginson relation [4] and the Roelands viscosity pressure relation [15] is used. The film thickness equation is made dimensionless using the same parameters and accounting for a moving surface feature reads:

$$
\begin{array}{r}
H(X, T)=H_{0}(T)-\mathcal{R}_{1}(X, T)-\mathcal{R}_{2}(X, T) \\
+\frac{X^{2}}{2}-\frac{1}{\pi} \int_{X_{\mathrm{a}}}^{X_{b}} P\left(X^{\prime}, T\right) \ln \left|X-X^{\prime}\right| d X^{\prime}
\end{array}
$$

where $\mathcal{R}_{1}(X, T)$ denotes the undeformed geometry of the waviness on the lower body at dimen- sionless time $T$ and $\mathcal{R}_{2}(X, T)$ describes the waviness on the upper body. $H_{0}(T)$ is an integration constant.

$$
\begin{aligned}
\mathcal{R}_{i}(X, T) & = \begin{cases}0, & X \geq X_{d, i} \\
\mathcal{A}_{i} \sin \left(2 \pi \frac{X-X_{d, i}}{\mathcal{W}_{i}}\right), & X<X_{d, i}\end{cases} \\
X_{d, i} & =X_{s}+2 u_{i} / u_{s} T ; \quad i=1,2
\end{aligned}
$$

At all times the force balance condition is imposed, i.e. the integral over the pressure must balance the externally applied contact load. This condition determines the value of the integration constant $H_{0}(T)$ in equation (2). Expressed in the dimensionless variables the force balance equation reads:

$$
\int_{X_{a}}^{X_{b}} P(X, T) d X-\frac{\pi}{2}=0 \quad \forall T
$$

\begin{tabular}{|l|l|l|}
\hline Parameter & Value & Dimension \\
\hline$E^{\prime}$ & $2.2610^{11}$ & {$[\mathrm{~Pa}]$} \\
\hline$\alpha$ & $2.210^{-8}$ & {$\left[\mathrm{~Pa}^{-1}\right]$} \\
\hline$z$ & 0.68 & \\
\hline$\eta_{0}$ & $4010^{-3}$ & {$[\mathrm{Pas}]$} \\
\hline$u_{s}$ & 1.94 & {$\left[\mathrm{~ms}^{-1}\right]$} \\
\hline$R$ & $1.4110^{-2}$ & {$[\mathrm{~m}]$} \\
\hline$b$ & $5.010^{-4}$ & {$[\mathrm{~m}]$} \\
\hline$p_{h}$ & $2.010^{9}$ & {$[\mathrm{~Pa}]$} \\
\hline$h_{\min }$ & 0.232 & {$[\mu \mathrm{m}]$} \\
\hline \hline \multicolumn{3}{|c|}{ Dimensionless parameters } \\
\hline$M$ & 100 & \\
\hline$L$ & 11.08 & \\
\hline \hline $\bar{\alpha}$ & 44.0 & \\
\hline$\lambda$ & $3.710^{-4}$ & \\
\hline \hline$W$ & $4.9210^{-4}$ & \\
\hline$U$ & $1.2210^{-11}$ & \\
\hline$G$ & $4.9710^{3}$ & \\
\hline \hline$H_{\min }$ & $1.31610^{-2}$ & \\
\hline$H_{a v e}$ & $1.48410^{-2}$ & \\
\hline$H_{\text {inv }}$ & $2.67410^{-2}$ & \\
\hline
\end{tabular}

Table 1: Different parameters and their values for the present loading condition.

\subsection{Conditions}

The parameters used to describe the contact conditions in the calculations are given in Table 1, to- 
gether with some dimensionless parameters. The numerical parameters used in the calculation are given in Table 2. These contact conditions are identical to the ones used in [19]. In that paper characteristics of the Reynolds equation are extensively described, especially the dominance of the shear flow over the pressure flow in the contact region. As a consequence the pressure perturbations become detached from the film thickness variations. For an extensive theoretical and numerical description of this effect the reader is referred to [19].

\begin{tabular}{|l|l|}
\hline Parameter & Value \\
\hline$X_{a}$ & -2.5 \\
\hline$X_{b}$ & 1.5 \\
\hline$\Delta_{T}$ & 0.005 \\
\hline$\Delta_{X}$ & $2.841 \quad 10^{-3}$ \\
\hline$n$ & 1409 \\
\hline
\end{tabular}

Table 2: Different parameters in the numerical solution.

\subsection{Time dependence}

Because we are dealing with a time dependent problem, the familiar stationary contact quantities like $H_{\min }, H_{a v e}$ and $P_{\max }$ now need another index to determine their relation with time. The superscript ..+ denotes the maximum value over time, the superscript ..- denotes the minumum value over time, and the superscript.- denotes the average value over time. These minima, maxima and averages are taken over the time interval when the values have become periodic, from $T=4.0$ onwards in case of figure 1 . This to exclude the initial period when the waviness is entering the contact area. The most interesting quantities are probably $H_{\min }^{-}$denoting the minimum value of the minimum film thickness over time, $\overline{H_{a v e}}$, the average value of the average film thickness over time and $P_{m a x}^{+}$the maximum value of the maximum pressure over time.

\subsection{Numerical accuracy}

In order to obtain a reasonable numerical accuracy for the extreme values like $H_{\min }^{-}$a fine mesh had to be combined with a small time increment.
The reader is reminded that $H_{\min }^{-}$is the minimum value over time of the minimum film thickness $H_{m i n}$. The numerical accuracy in this quantity is better than $10 \%$ over the entire parameter range presented. The average film thicknesses $\overline{H_{a v e}}$ and $\widetilde{H}_{i n v}$ converge much more rapidly. Generally, the numerical error in these quantities is less than $2 \%$. In order to eliminate the start-up effects and to obtain results from a fully periodic solution, the number of time steps was chosen inversely proportional to the velocity of the slowest surface. Thus 4000 timesteps were used for $u_{2} / u_{s}=0.1$ and $u_{2} / u_{s}=0.9,2000$ timesteps were used for $u_{2} / u_{s}=0.2$ and $u_{2} / u_{s}=0.8$, 1500 timesteps were used for $u_{2} / u_{s}=0.3$ and $u_{2} / u_{s}=0.7$ and 1000 timesteps were used for $u_{2} / u_{s}=0.4$ and $u_{2} / u_{s}=0.6$.

\section{General results}

In figures 1, 2 and 3, three different ways of presenting the solutions are shown. Each has its own advantages and disadvantages, and each one sheds light on a particular detail of the results The conditions were $w=0.25 b, A=0.25 \mu \mathrm{m}$, $u_{2} / u_{s}=0.25$ (thus $u_{1} / u_{s}=0.75$ and $u_{1} / u_{2}=$ $3.0), X_{d, 1}=X_{d, 2}=-2.0$ at $T=0.0$. Figure 1 shows the pressure $(P)$ and film thickness $(H)$ at a location $(X=0)$ as a function of the dimensionless time $(T)$. At $T=0$ the waviness on both surfaces is outside the contact and both pressure and film thickness have their smooth surface values. From $T=2 u_{s} /\left(2 u_{1}\right)=1.33$ onwards the waviness of the lower (faster) body reaches $X=0.0$ and the pressure variations become sinusoidal. From $T=2 u_{s} /\left(2 u_{2}\right)=4.0$ onwards the upper body waviness reaches this location and the pressure becomes harmonic. Because the film thickness variations generated by the waviness of the upper and lower body travel at an average surface velocity $\left(u_{s} / 2\right)$ within the high pressure region $(|X|<1.0)$ the two variations in the film thickness reach $X=0.0$ at $T=u_{s} /\left(2 u_{2}\right)+1.0=3.0$ and $T=u_{s} /\left(2 u_{1}\right)+1.0=1.66$ respectively. As a consequence the pressure and film thickness variations shown in figure 1 become harmonic at different times $(T=4.0$ and $T=3.0$ respectively). For locations $X>0$ this time difference is even greater. 


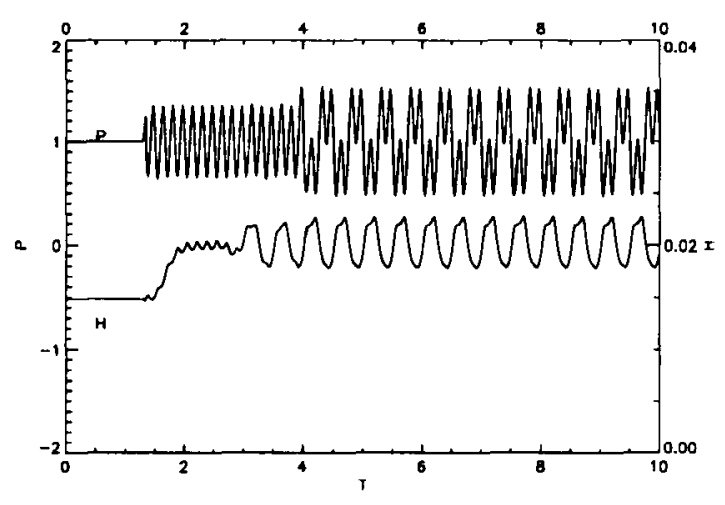

Figure 1 Pressure and film thickness for $X=0$ as a function of $T, u_{2} / u_{s}=0.25$

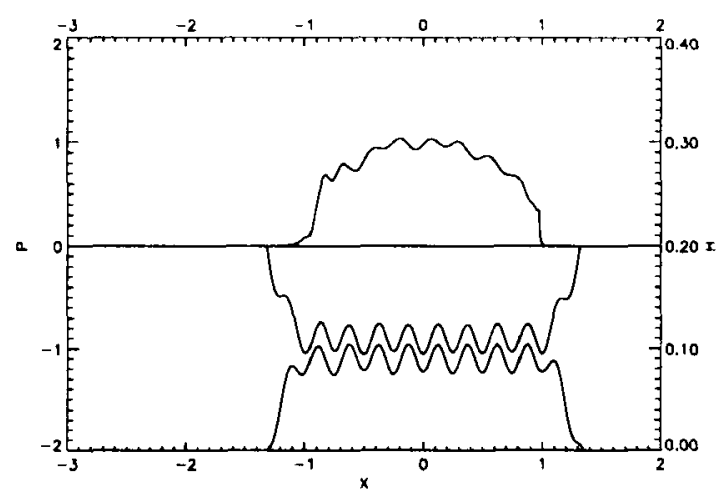

Figure 2a Pressure and film thickness for $T=$ 6.88 as a function of $X, u_{2} / u_{s}=0.25$

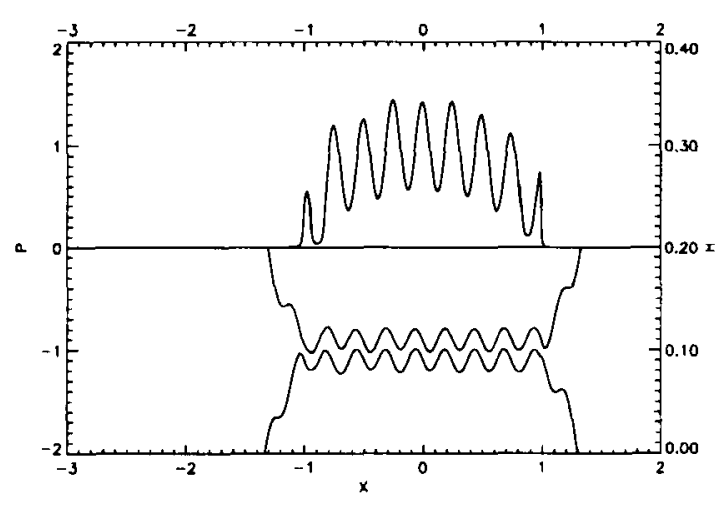

Figure 2b Pressure and film thickness for $T=$ 6.94 as a function of $X, u_{2} / u_{s}=0.25$

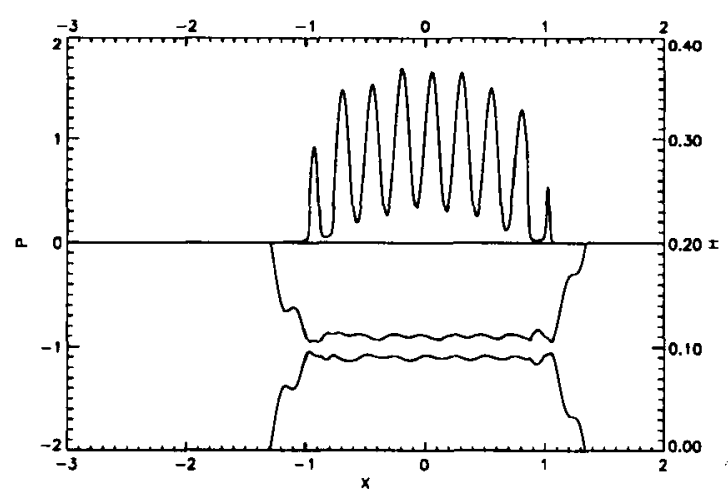

Figure 2c Pressure and film thickness for $T=$ 7.00 as a function of $X, u_{2} / u_{s}=0.25$

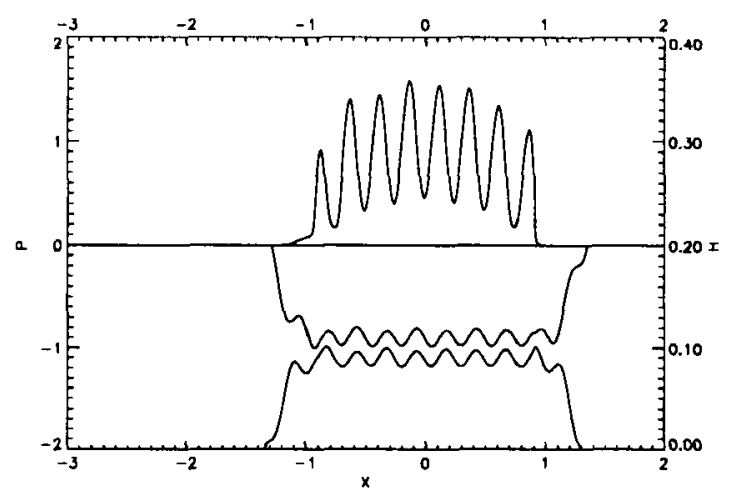

Figure 2d Pressure and film thickness for $T=$ 7.06 as a function of $X, u_{2} / u_{s}=0.25$

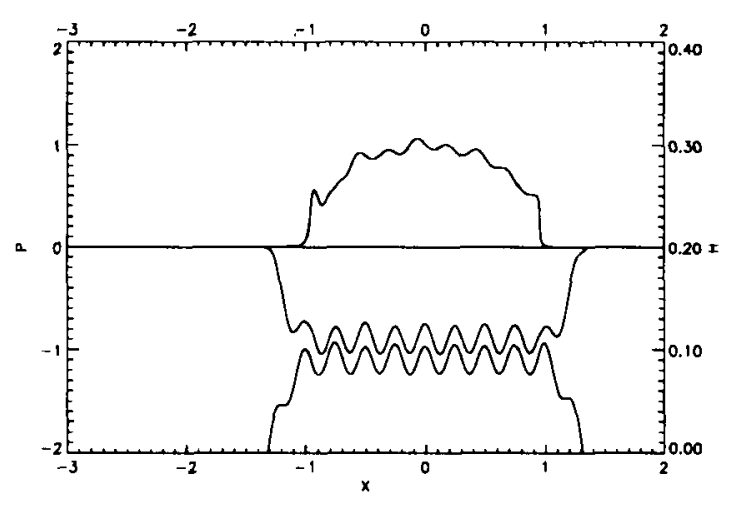

Figure 2e Pressure and film thickness for $T=$ 7.13 as a function of $X, u_{2} / u_{s}=0.25$ 
A second way of visualizing the data is shown in figures 2a-e which displays the pressure and film thickness profiles for a certain timestep $(T=6.88,6.94,7.00,7.06,7.13)$ as a function of $\mathrm{X}$. From these figures it is clear that the maximum pressure fluctuations coincide with the minimum film thickness variations, and vice versa. Because the lower body velocity is three times larger than the upper body velocity the lower body waviness has to "jump over" the upper body waviness, thereby causing a major flattening of the waviness and therefore requiring the large pressure fluctuations (figure $2 \mathrm{c}$ ). The pressure fluctuations are minimal when the waviness of both surfaces is in phase (figure $2 \mathrm{a}$ and $2 \mathrm{e}$ ). Clearly, this type of data is better presented as a movie.

The third way of presenting the pressure and film thickness variations is by considering them as a function of time for a certain location on the bodies. This description is important when one is interested in the stresses and stress cycles a certain volume element inside the material experiences. Figure 3 a displays film thickness and pressure on a waviness top located on the upper (slow) surface, figure $3 \mathrm{~b}$ does the same for a waviness top on the lower (fast) surface. Note that the time spent in the contact zone for the upper (slow) surface is three times as large as for the lower (fast) surface.

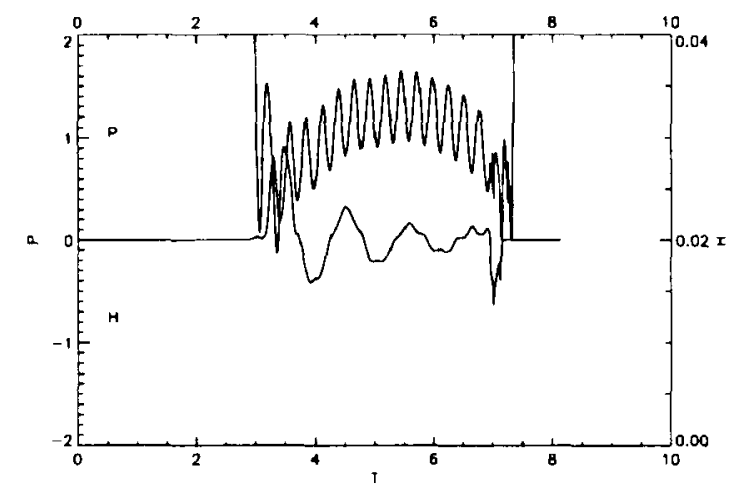

Figure 3a Pressure and film thickness for a waviness top on the upper surface as a function of $T$, $u_{2} / u_{s}=0.25$

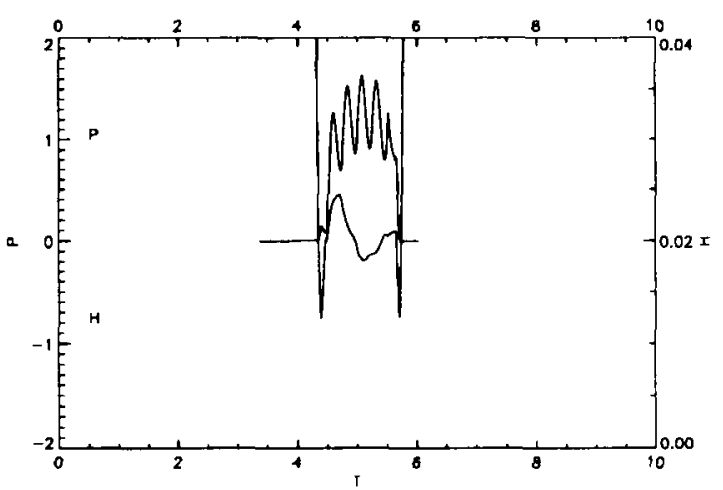

Figure $3 \mathbf{b}$ Pressure and film thickness for a waviness top on the lower surface as a function of $T$, $u_{2} / u_{s}=0.25$

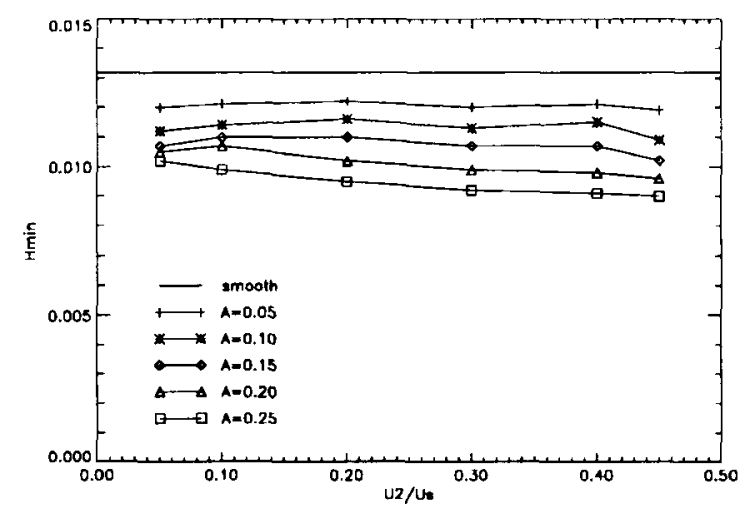

Figure 4a Minimum film thickness $H_{\min }^{-}$as a function of $u_{2} / u_{s}, A_{1}=A_{2}=A$ in $\mu \mathrm{m}, \mathcal{W}_{1}=$ $\mathcal{W}_{2}=0.25$

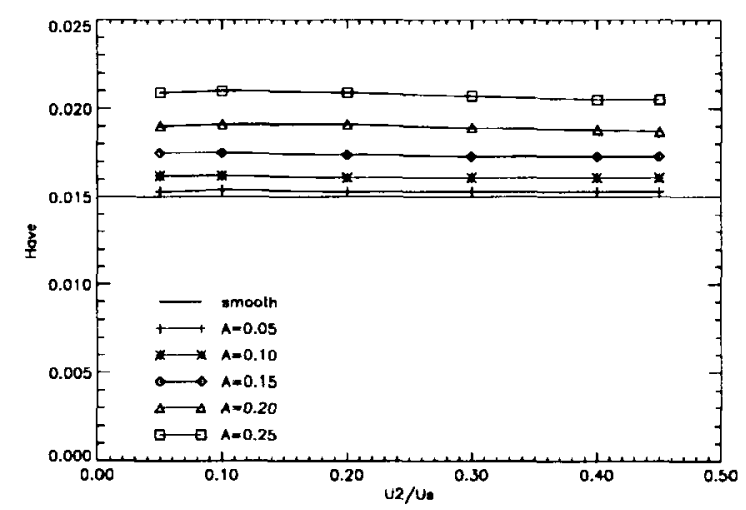

Figure $4 \mathbf{b}$ A verage film thickness $\overline{H_{\text {ave }}}$ as a function of $u_{2} / u_{s}, A_{1}=A_{2}=A$ in $\mu \mathrm{m}, \mathcal{W}_{1}=\mathcal{W}_{2}=$ 0.25 


\section{Influence of $u_{2} / u_{s}$ for equal waviness amplitude}

From figure 1 it can be observed that the film thickness at $X=0.0$ increases over its smooth value when the waviness enters the contact. This section explores how the different parameters like minimum film thickness, average film thickness and maximum pressure behave as a function of the surface speeds. Figures $4 \mathrm{a}$ and $\mathrm{b}$ show the influence of the parameter $u_{2} / u_{s}$ on the minimum film thickness and on the average film thickness for the case that the waviness on both surfaces has the same amplitude. The definitions of $H_{\min }^{-}$and $\overline{H_{a v e}}$ are given in section 2.3. Only the first half of the graphs is shown since they are symmetric around $u_{2} / u_{s}=0.5$. The points $u_{2} / u_{s}=0.0$ and 0.5 are omitted since they do not represent an average over all phase differences of the waviness.

From these figures it can be observed that the minimum film thickness decreases with amplitude below the smooth surface value. The average film thickness increases with increasing amplitude over the smooth surface value. From these two figures it can also be concluded that the influence of the surface velocity difference on the minimum and average film thickness is small. Also the maximum pressure was found to be insensitive to the velocity difference.

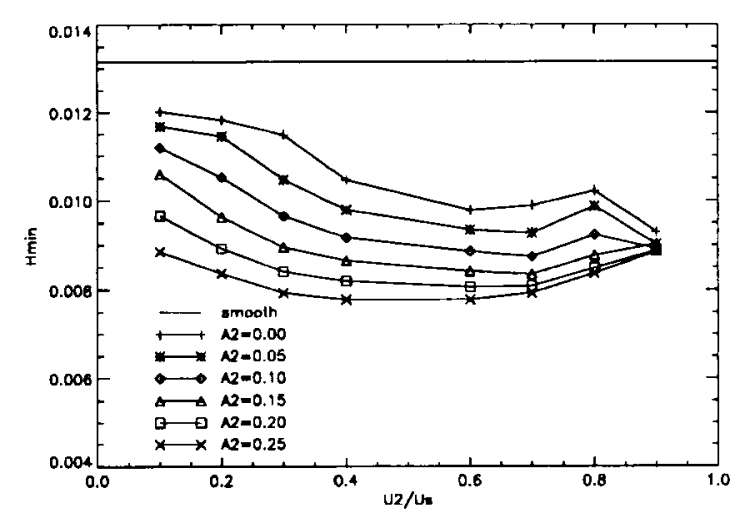

Figure 5a Minimum film thickness $H_{\min }^{-}$as a function of $u_{2} / u_{s}$ and $A_{2}, A_{1}=0.25 \mu \mathrm{m}, \mathcal{W}_{1}=$ $\mathcal{W}_{2}=0.25$

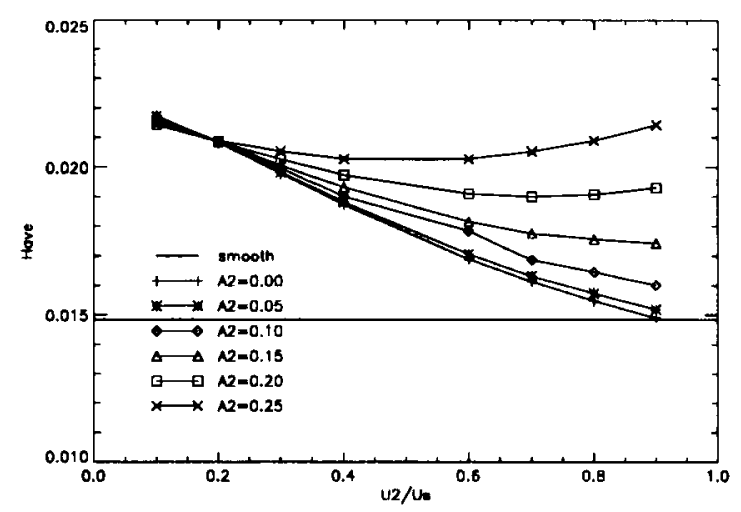

Figure 5b A verage film thickness $\overline{H_{\text {ave }}}$ as a function of $u_{2} / u_{s}$ and $A_{2}, A_{1}=0.25 \mu \mathrm{m}, \mathcal{W}_{1}=\mathcal{W}_{2}=$ 0.25

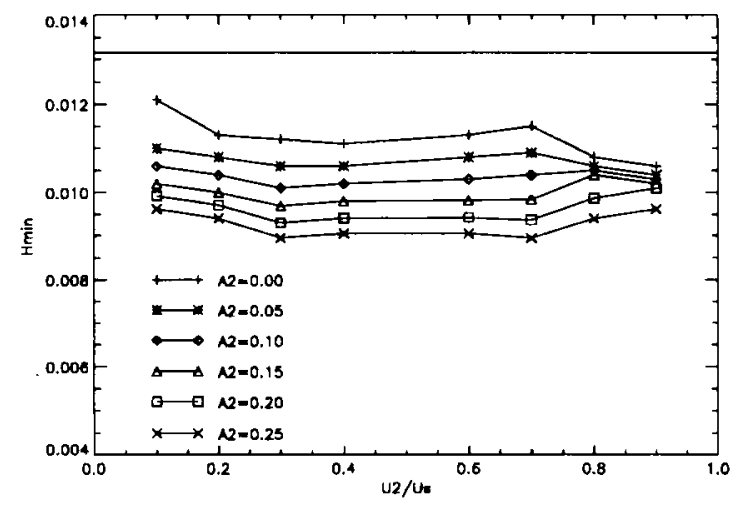

Figure 6a Minimum film thickness $H_{\min }^{-}$as a function of $u_{2} / u_{s}$ and $A_{2}, A_{1}=0.25 \mu \mathrm{m}, \mathcal{W}_{1}=$ $\mathcal{W}_{2}=0.50$

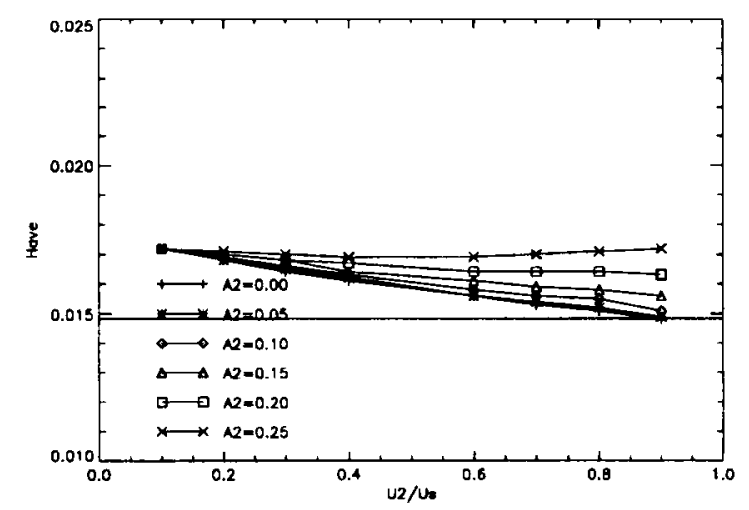

Figure $6 \mathrm{~b}$ A verage film thickness $\overline{H_{\text {ave }}}$ as a function of $u_{2} / u_{s}$ and $A_{2}, A_{1}=0.25 \mu \mathrm{m}, \mathcal{W}_{1}=\mathcal{W}_{2}=$ 0.50 


\section{Influence of $u_{2} / u_{s}$, for dif- ferent waviness amplitude}

In this section the waviness amplitude of the lower surface is kept contant $A_{1}=0.25$, while the amplitude of the upper body waviness is varied from $A_{2}=0.0$ to $A_{2}=0.25$. Now the symmetry around $u_{2} / u_{s}=0.5$ is broken of course, and the entire range from $u_{2} / u_{s}=0.1$ to $u_{2} / u_{s}=0.9$ is displayed. The points $u_{2} / u_{s}=0.0,0.5$ and 1.0 are again omitted since they do not represent an average over all phase differences.

From figure $5 \mathrm{a}$ it can be observed that the minimum film thickness decreases with increasing amplitude $A_{2}$ and with increasing values of $u_{2} / u_{s}$ as was found in the previous section. For large values of $u_{2} / u_{s}$ and $A_{2}$ it increases slightly again, in order to become symmetric for the case of $A_{2}=0.25$. From figure $5 \mathrm{~b}$ it can be observed that the average film thickness increases with increasing amplitude $A_{2}$ and decreases with increasing values of $u_{2} / u_{s}$. For large values of $u_{2} / u_{s}$ and $A_{2}$ it increases slightly again, becoming symmetric around $u_{2} / u_{3}=0.5$ for the case that $A_{1}=A_{2}=0.25$. The maximum pressure $P_{\max }^{+}$was found to be virtually independent of the value of $u_{2} / u_{s}$, as was observed in the previous section.

In figure 6 the wavelength was doubled compared to figure 5. The influence of this doubling on the minimum film thickness is small, compare figures $5 \mathrm{a}$ and $6 \mathrm{a}$. The maximum pressure is halved for a given amplitude, as is predicted by the dry contact theory. The increase of the average film thickness over its smooth value and its dependency on $u_{2} / u_{s}$ and $\mathcal{A}_{2}$ are considerably reduced as can be observed by comparing the figures $5 \mathrm{~b}$ and $6 \mathrm{~b}$. Roughly speaking, the increase in average film thickness is halved by doubling the wavelength of the waviness.

\section{Influence of amplitude}

This section analyses in detail the influence of the amplitude on the maximum pressure, the minimum and the average film thickness as observed in the previous section. From a dry contact analysis the maximum pressure can be expected to increase linearly with the waviness amplitude, a

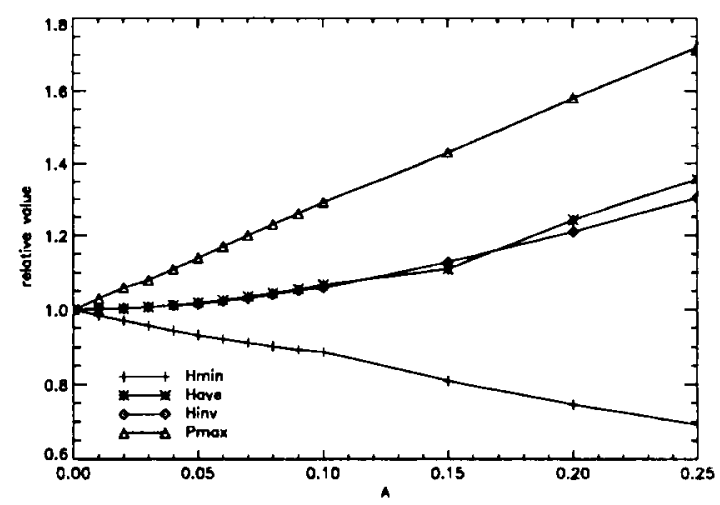

Figure 7a Relative change in pressure $P_{\max }^{+}$and film thickness $H_{\min }^{-}, \overline{H_{i n v}}$ and $\overline{H_{a v e}}$ as a function of the amplitude $A_{1}=A_{2}=A$ in $\mu \mathrm{m}, \mathcal{W}_{1}=$ $\mathcal{W}_{2}=0.25, u_{2} / u_{s}=0.3$

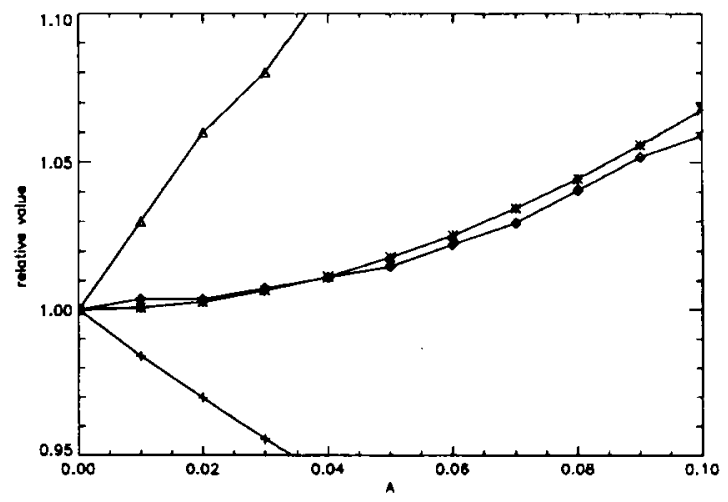

Figure 7b Detail of figure $7 \mathbf{a}$

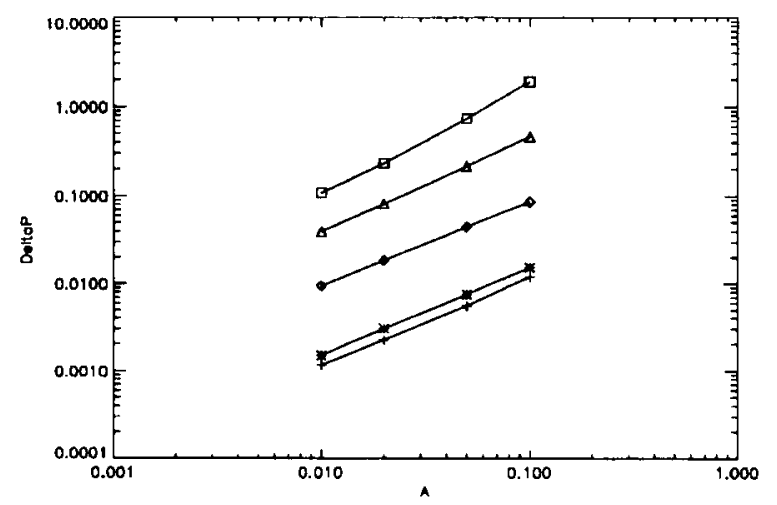

Figure 8 Relative pressure increase in the inlet region as a function of the amplitude $A_{1}=A_{2}=$ $A$ in $\mu \mathrm{m}, \mathcal{W}_{1}=\mathcal{W}_{2}=0.25, u_{2} / u_{s}=0.3$, bottom to top for $X=-2.0,-1.5,-1.2,-1.1,-1.0$. 
trend that is observed from figure $7 \mathbf{a}$ and is analysed in detail in [7]. The minimum film thickness decreases roughly linear with the amplitude, a trend that is easily accepted. However, the average film thickness as well as the inverse film thickness seem to increase quadratically with the amplitude. This can be more clearly abserved from figure $7 \mathrm{~b}$, which displays a detail of figure $7 \mathrm{a}$. This trend, which was also observed in [19], needs further explanation.

The classical explanation of the smooth average film thickness and the nearly parallel film thickness in the contact area assumes that the pressure in the inlet reaches a certain value where the oil viscosity becomes so large that pressureinduced flow is nearly absent. From this (zeropressure-flow) point onwards, all oil is transported through the contact.

This explanation can be extended to wavy contacts. Consider a point in the inlet where the pressure is such that the viscosity becomes sufficiently high to eliminate virtually all pressure induced flow. Now assume that in the neighbourhood of this point the pressure fluctuations depend linearly on the waviness amplitude, as happens in the contact region (see figure $7 \mathrm{a}$ ). The location of this zero pressure-flow point then moves outwards in a first approximation proportionally with the height of the pressure fluctuations which are themselves proportional to the waviness amplitude. The gap height at this point increases roughly proportional with the square of this distance, since the cylinder can be accurately approximated by a parabola as the influence of deformation can be neglected. As a result the gap height at this point, and therefore the amount of oil transported through the contact, and thus the average film thickness in the contact area will increase with the square of the waviness amplitude. Figure 8 shows the relative increase of the maximum pressure in the inlet at various locations as a function of the amplitude. From this figure it can be concluded that the pressure increase in the inlet is indeed proportional to the waviness amplitude, as is the case in the Hertzian contact zone. Concluding this section it can be stated that the average film thickness increases quadratically with the waviness amplitude because the location of the zero-pressure-flow point moves lin- early towards the inlet as a function of the waviness amplitude.

\section{Discussion and conclusion}

In this paper the influence of two-sided waviness on pressure and film thickness was investigated. The influence of the non-Newtonian fluid behaviour as well as thermal effects has been omitted. The authors are aware of the importance of these effects especially at large velocity differences. However, in order to be able to correctly interpret the results it was considered beneficial to study one effect at a time.

The observed dependence of $P_{\max }^{+}, H_{\min }^{-}$and $\overline{H_{\text {ave }}}$ on $\mathcal{A}_{1}, \mathcal{A}_{2}, \mathcal{W}_{1}, \mathcal{W}_{2}$ and $u_{2} / u_{s}$ can be summarized as folows. The parameters with the additional subscript "s" refer to the parameter values for the smooth solution.

- The maximum pressure attained in the oil film is close to the dry contact pressure, is virtually independent of the ratio of the surface velocities and increases linearly with the ratio of amplitude and wavelength:

$P_{\text {max }}^{+}=P_{\text {max }, s}+c_{1}\left(\mathcal{A}_{1} / \mathcal{W}_{1}+\mathcal{A}_{2} / \mathcal{W}_{2}\right)$.

- The minimum film thickness $H_{m i n}^{-}$attained in the oil film decreases linearly with increasing waviness amplitude and is independent of the wavelength $\mathcal{W}$. As a first approximation:

$H_{\min }^{-}=H_{\min , s}-c_{2}\left(\mathcal{A}_{1} u_{1}+\mathcal{A}_{2} u_{2}\right)$.

- The average film thickness $\overline{H_{a v e}}$ attained in the oil film increases quadratically with ratio of amplitude and wavelength for small amplitudes. As a first approximation:

$\overline{H_{\text {ave }}}=H_{\text {ave }, s}+c_{3}\left(\mathcal{A}_{1} u_{1} / \mathcal{W}_{1}+\mathcal{A}_{2} u_{2} / \mathcal{W}_{2}\right)^{2}$.

\section{Acknowledgement}

The authors would like to thank Dr. H.H. Wittmeyer, Managing Director of the SKF Engineering \& Research Centre for his kind permission to publish this work, part of which was supported by a fellowship of the "Royal Netherlands Academy of Arts and Sciences". 


\section{References}

[1] Chang, L., Cusano, C., and Conry, T.F., 1989, "Effects of Lubrication Rheology and Kinematic Conditions on MicroElastohydrodynamic Lubrication," $A S M E$ JOT, 111, 344-351.

[2] Chang, L., and Webster, M.N., 1991, "A Study of Elastohydrodynamic Lubrication of Rough Surfaces," ASME JOT, 113, 110-115.

[3] Chang, L., 1992, "Traction in Thermal Elastohydrodynamic Lubrication of Rough Surfaces," ASME JOT, 114, 186-191.

[4] Dowson, D., and Higginson, G.R., 1966, "Elastohydrodynamic Lubrication, The Fundamentals of Roller and Gear Lubrication," Pergamon Press, Oxford, Great Britain.

[5] Goglia, P.R., Cusano, C., and Conry, T.F., 1984, "The Effects of Surface Irregularities on the Elastohydrodynamic Lubrication of Sliding Line Contacts. Part I - Single Irregularities," ASME JOT, 106, 104-112.

[6] Goglia, P.R., Cusano, C., and Conry, T.F., 1984, "The Effects of Surface Irregularities on the Elastohydrodynamic Lubrication of Sliding Line Contacts. Part II - Wavy Surfaces," ASME JOT, 106, 113-119.

[7] Greenwood, J.A., and Johnson, K.L. 1992, "The Behaviour of Transverse Roughness in Sliding Elastohydrodynamically Lubricated Contacts," WEAR, 153, 107-117.

[8] Kaneta, M., 1991, "private communication"

[9] Kweh, C.C., Evans, H.P., and Snidle, R.W., 1989, "Micro-Elastohydrodynamic Lubrication of an Elliptical Contact with Transverse and Three-Dimensional Roughness," ASME JOT, 111, 577-583.

[10] Lee, R.T., and Hamrock, B.J., 1990, "A circular non-Newtonian Fluid Model: Part II - used in Micro-Elastohydrodynamic Lubrication," ASME JOT, 112, 497-505.
[11] Lubrecht, A.A., Venner, C.H., Lane, S., Jacobson, B., and Ioannides, E., 1990, "Surface Damage - Comparison of Theoretical and Experimental Endurance Lives of Rolling Bearings," Proceedings of the 1990 Japan International Tribology Conference, Nagoya, Japan, 1, 185-190.

[12] Lubrecht, A.A., 1987, "Numerical Solution of the EHL Line and Point Contact Problem Using Multigrid Techniques," PhD. Thesis, University of Twente, Enschede, The Netherlands. ISBN 90-9001583-3

[13] Lubrecht, A.A., ten Napel, W.E., Bosma, R., 1988, "The Influence of Longitudinal and Transverse Roughness on the Elastohydrodynamic Lubrication of Circular Contacts", ASME JOT, 110, 421-426.

[14] Osborn, K.F., Sadeghi, F., 1992, "Time Dependent Line EHD Lubrication using the Multigrid/Multilevel Technique", $A S M E$ JOT, 114, 68-74.

[15] Roelands, C.J.A., 1966, "Correlational Aspects of the Viscosity-TemperaturePressure Relationship of Lubricating Oils," PhD. Thesis, Technical University Delft, Delft, The Netherlands, (V.R.B., Groningen, The Netherlands).

[16] Venner, C.H., ten Napel, W.E., and Bosma, R., 1990, "Advanced Multilevel Solution of the EHL Line Contact Problem," ASME JOT, 112, 426-432.

[17] Venner, C.H., Lubrecht, A.A., and ten Napel, W.E., 1991, "Numerical Simulation of the Overrolling of a Surface Feature in an EHL Line Contact," ASME JOT, 113, 777 783 .

[18] Venner, C.H., 1991, "Multilevel Solution of the EHL Line and Point Contact Problems," PhD. Thesis, University of Twente, Enschede, The Netherlands. ISBN 90-9003974-0.

[19] Venner, C.H., and Lubrecht, A.A. 1992, "Transient Analysis of Surface Features in an EHL Line Contact in the case of Sliding," to appear in ASME JOT 